\title{
Circadian Control of the Pupillary Light Responses in an Avian Model of Blindness, the GUCY1* Chickens
}

\author{
Diego J. Valdez, ${ }^{*, 1}$ Paula S. Nieto,${ }^{\dagger, 1}$ Natalia S. Della Costa,${ }^{2}$ Clemar Schurrer, ${ }^{3}$ \\ and Mario E. Guido ${ }^{1}$
}

${ }^{1}$ Centro de Investigaciones en Química Biológica de Córdoba (CIQUIBIC-CONICET) Departamento de Química Biológica, Facultad de Ciencias Químicas, Universidad Nacional de Córdoba (UNC), Córdoba, Argentina

${ }^{2}$ Instituto de Diversidad y Ecología Animal (IDEA-CONICET), Centro de Zoología Aplicada, Universidad Nacional de Córdoba (UNC), Córdoba, Argentina

${ }^{3}$ CEMETRO, UTN-FRC, Córdoba, Argentina

Correspondence: Mario E. Guido, CIQUIBIC-Departamento de Química Biológica, Facultad de Ciencias Químicas, Universidad Nacional de Córdoba-CONICET, Haya de la Torre s/n, Ciudad Universitaria, 5000 Córdoba, Argentina; mguido@fcq.unc.edu.ar.

DJV and PSN contributed equally to the work presented here and should therefore be regarded as equivalent authors.

Current affiliation: *Instituto de Diversidad y Ecología Animal (IDEACONICET), Centro de Zoología Aplicada, Universidad Nacional de Córdoba, Córdoba 5000, Argentina. †Facultad de Matemática, Astronomía y Física (FaMAF), Instituto de Física Enrique Gaviola (IFEG-CONICET), Universidad Nacional de Cordoba, Cordoba, Argentina.

Submitted: August 15, 2014

Accepted: December 11, 2014

Citation: Valdez DJ, Nieto PS, Della

Costa NS, Schurrer C, Guido ME.

Circadian control of the pupillary light responses in an avian model of blindness, the GUCY1* chickens. Invest Ophthalmol Vis Sci. 2015;56:730-

737. DOI:10.1167/iovs.14-15481
Punpose. The vertebrate inner retina has a subset of intrinsically photosensitive retinal ganglion cells (ipRGCs) that express the nonvisual photopigment melanopsin. The intrinsically photosensitive retinal ganglion cells send light information from the environment to the brain to control, among other parameters, the amount of energy entering the eyes through the pupillary light reflex (PLR). A daily variation in the PLR in both mice and humans has recently been shown, indicating circadian control of this response. In a previous work involving the sensitivity spectra for the PLR, we showed that blind chickens (GUCY1*) display the highest sensitivity to light of $480 \mathrm{~nm}$. The aim of the present study was to evaluate the potential circadian control of PLRs in blind birds under scotopic conditions.

Methods. Circadian PLR was performed on GUCY1* chickens with lights of different wavelengths (white or blue light of $475 \mathrm{~nm}$ ) under scotopic conditions.

Results. We found a significant daily variation in the PLRs of chickens exposed to white or blue light of $475 \mathrm{~nm}$, with increased sensitivity at circadian time 6 during the subjective day.

Conclusions. Our observations clearly point to circadian control of PLRs even in blindness, strongly indicating that both the entry of light into the eyes and its quality are differentially regulated during the day in diurnal animals.

Keywords: pupillary light reflexes, melanopsin, blindness, circadian rhythm, nonvisual phototransduction

\begin{abstract}
Tight detection by the inner retina (inner nuclear and Langlion cell layer) has been demonstrated in several vertebrate species. ${ }^{1-6}$ This capacity is given by a subset of intrinsically photosensitive ganglion cells (ipRGCs) expressing the photopigment melanopsin. ${ }^{7}$ These cells project and send light information to different brain areas such as the suprachiasmatic nucleus, the pretectal olivary nucleus and the lateral geniculate nucleus, among others. ${ }^{8}$ The information sent to the brain by the ipRGCs serves to control several nonvisual tasks such as the synchronization of circadian rhythms, the photic suppression of pineal melatonin, and the control of direct and consensual pupillary light reflexes ( ${ }_{d} P L R$ and $\left.{ }_{c} P L R\right) .{ }^{6,9-12}$ The pupillary light reflex regulates the amount of light entering the eye and thus reaching the retina. Under continuous light conditions, both the direct and consensual PLRs exhibit two
\end{abstract}

Copyright 2015 The Association for Research in Vision and Ophthalmology, Inc. www.iovs.org | ISSN: 1552-5783 clearly distinguishable stages: the nonstationary phase at first and then the stationary phase at the end. In the nonstationary phase, an immediate pupil constriction is observed (usually commanded by classical photoreceptors, rods and cones), ${ }^{13}$ giving an idea of the kinetics of the constriction process, whereas in the stationary phase, a steady-state pupil size is visualized in which all the retinal photosensitive elements (classical photoreceptors and ipRGCs) participate. ${ }^{13}$

Recently, daily differences in the mouse and human PLRs have been reported, suggesting the existence of circadian control of the PLR in these mammalian species. ${ }^{2,14}$ Previously we demonstrated that the inner retina of blind chickens (GUCY1*) is able to perceive light and conduct a number of nonvisual tasks such as the photic entrainment of food intake rhythms and pupillary light constriction. ${ }^{6}$ According to the 
action/sensitivity spectra assessed, these animals have the highest sensitivity to light of $480 \mathrm{~nm}$ for the consensual PLRs; this closely resembles the two melanopsin (Opn4) photopigments, Opn $4 x$ and Opn $4 m$, Xenopus and mammalian orthologs respectively, whose mRNAs and proteins were shown to be expressed in the inner retina of GUCY1* chickens. ${ }^{6,15}$ Given this background, the aim of this work was to evaluate the potential circadian control of the consensual PLRs (nonstationary and stationary phases) in GUCY1* chickens under scotopic conditions with different types of light: white (including the whole visible spectra) and blue of $475 \mathrm{~nm}$ corresponding to the maximal absorbance for melanopsin photopigments. ${ }^{6}$

\section{Materials AND Methods}

\section{Animal Handling}

Blind chicks (GUCY1*, $n=3$ ) aged 3 to 4 months were maintained in light-dark cycles (LD; 600 lux, cool white fluorescent light) of 12 hours, each with food and water ad libitum and a room temperature of $25^{\circ} \mathrm{C}$. The animals were subsequently released to constant darkness (DD) for 3 days, during which animal handling was carried out under dim red light ( $<3$ lux). Lights used were standard fluorescent lights: Sylvania (Erlangen, Germany), F18 W/154-T8, daylight, recyclable, made in Germany, with the emission spectrum ranging from 390 to $627 \mathrm{~nm}$. Animal handling was performed according to the Guide to the Care and Use of Experimental Animals published by the Canadian Council on Animal Care and approved by the local animal care committee (School of Chemistry, National University of Cordoba, Exp. 15-99-39796). All ocular procedures were performed according to the ARVO Statement for the Use of Animals in Ophthalmic and Vision Research.

\section{Light Stimulation}

Light stimuli were provided by a white light ophthalmologic lantern or an ophthalmologic lantern adapted with two types of LEDs, one of $475 \mathrm{~nm}$, and the other of $430 \mathrm{~nm}$ as a control. Light intensity was determined with a silicon photodiode (data logging light meter, model 401036; Extech Instruments, Waltham, MA, USA) and the light intensities used were 3000 lux for white light, $\approx 150 \operatorname{lux}\left(12.05 \mathrm{~mW} / \mathrm{cm}^{2}\right)$ for $475 \mathrm{~nm}$, and $\approx 60 \operatorname{lux}\left(0.35 \mathrm{~mW} / \mathrm{cm}^{2}\right)$ for controls at $430 \mathrm{~nm}$.

\section{Pupillometry}

Pupillometry was undertaken in non-anesthetized GUCY1* chicks $(n=3)$ as previously described in Valdez et al. ${ }^{6}$ Animals were previously adapted to LD cycles for 15 days and then released to DD for 3 days. On the third day in DD, measurements were carried out at different circadian times. One eye (consensual) of each animal was photographed under dim red light with a digital camera (Nikon Co-olpix, 3.2 megapixel, Nikon Corp., Tokyo, Japan), and serial pictures were taken for 32 seconds after light exposure of different lights (white and 475-nm blue light ). A light control of $430 \mathrm{~nm}$ was also included. The interval between flashes was from 5 to 10 minutes, the time taken for the animals to become darkadapted.

\section{Definition and Estimation of Nonstationary and Stationary Phases}

The pupillary light reflex has two clearly distinguishable phases, nonstationary (NSP) and stationary (SP), which provide different but complementary information.

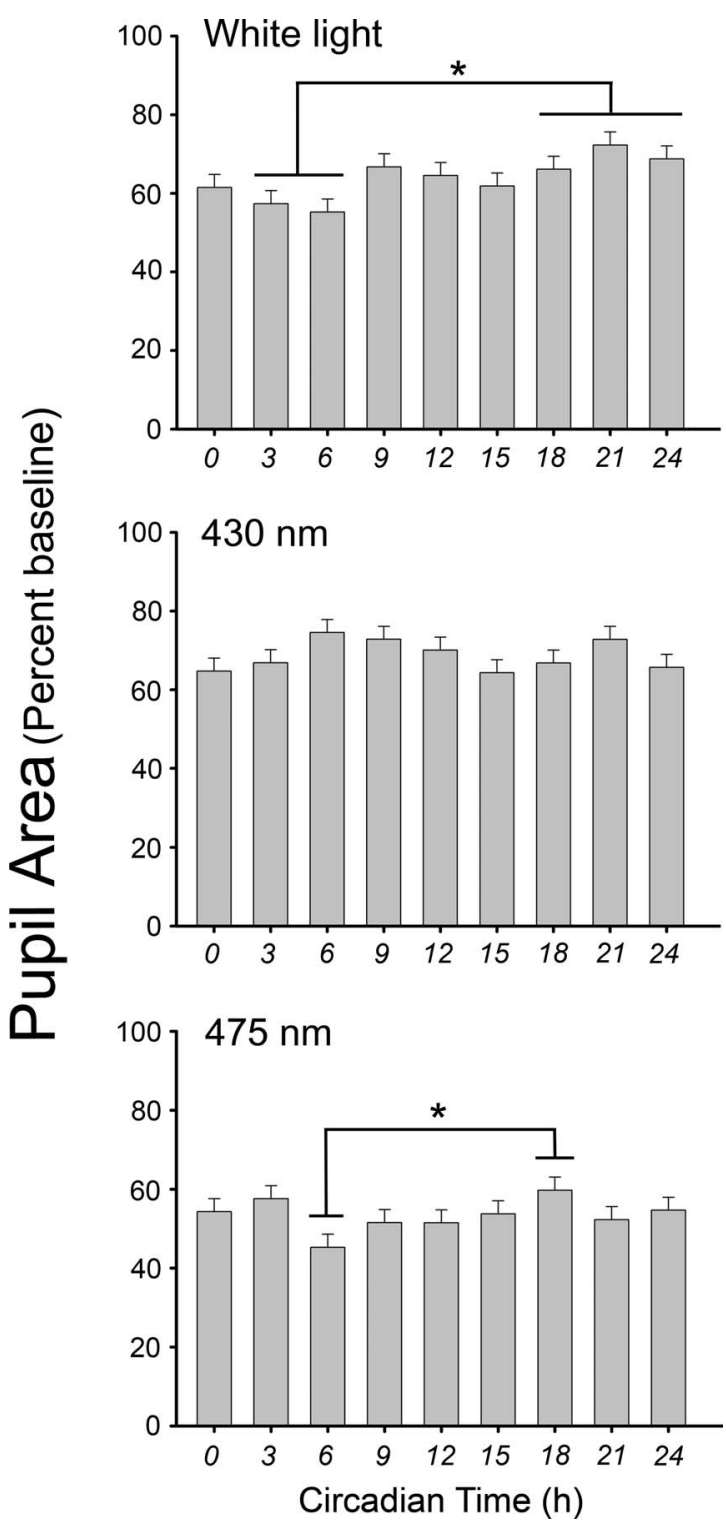

FIgURE 1. Circadian pupillary light response (SP and NSPs) in GUCY1* chickens exposed to different types of light under scotopic conditions (test 1). Upper: circadian variation of PLR with bright white light. Middle: pupillary light reflexes with blue light of $430 \mathrm{~nm}$, Bottom: circadian variation of PLR with blue light of $475 \mathrm{~nm}$. Results are presented as percentage of maximum linear pupil constriction. Data are means $\pm \mathrm{SEM} ; n=3$. Statistical analysis revealed a significant effect of time of day for white and blue light (475 nm; $P \leq 0.0001)$.

The nonstationary phase is defined as the interval of time in which the pupil size contracts toward its final size and provides information about the pupil constriction kinetics (we study the characteristic lifetime ( $\tau$, sec) for the PRL time courses throughout the day). In our experiments, the NSP begins when the light stimulus is switched on and ends when the pupil size reaches its steady minimum size, after 18 seconds of stimulation. The pupil remains at this steady minimum size for as long as the light stimulus lasts, which defines the SP in this study from 18 to 32 seconds of stimulation.

\section{Statistics}

Statistical analyses were performed using the InfoStat statistical software package (InfoStat, Grupo InfoStat, FCA-UNC, Argenti- 

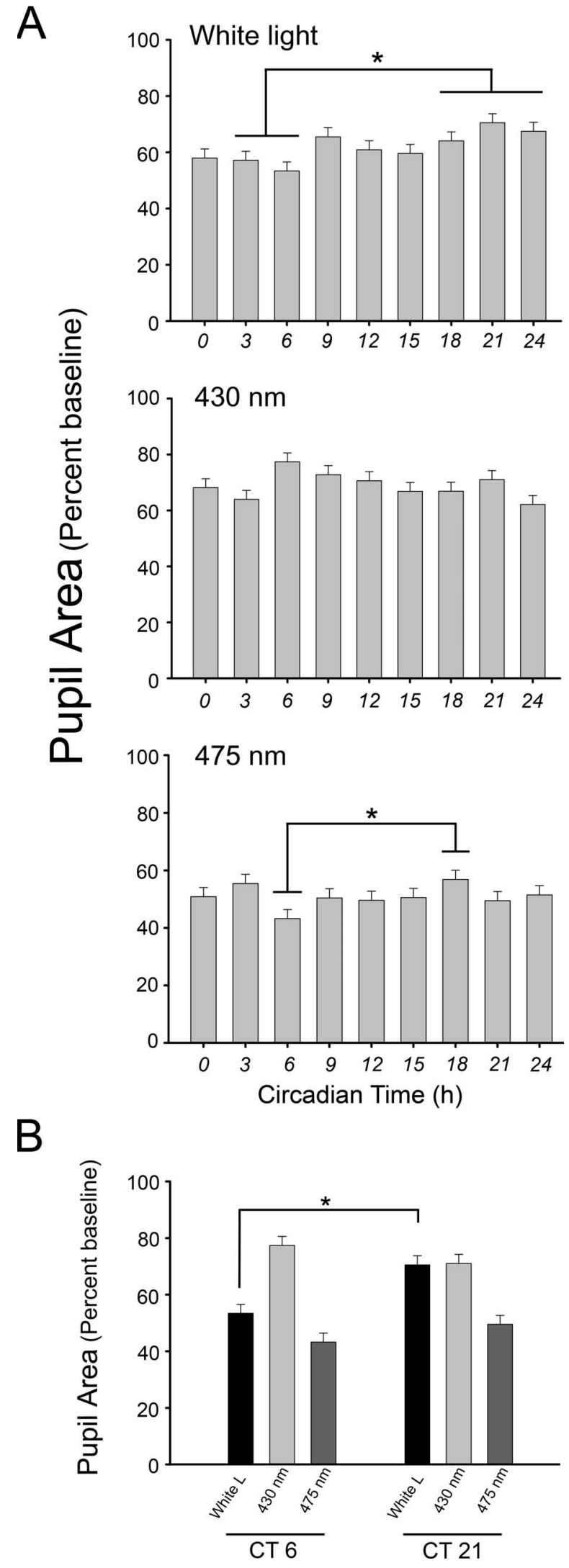

Figure 2. Analysis of the nonstationary pupillary light responses in GUCY1* chickens exposed to different types of light under scotopic conditions. (A) Circadian pupillary light response at NSP (0-18 seconds of stimulation) in GUCY1* chickens exposed to different types of light under scotopic conditions (test 2). Upper: circadian na). ${ }^{16}$ The pupillary light reflex data were tested by means of mixed-model statistical analysis to evaluate the effects of circadian time, type of light, stimulation time, and the interaction among these factors. Pupillary light reflex was considered a dependent variable. We included circadian time, type of light, and stimulation time as fixed factors and animal as random effect (test 1).

We conducted a separated analysis of our results for these two phases (NSP and SP).

The pupillary light reflex data from the NSP were compared by mixed-model statistical analysis that evaluated the effects of circadian time, type of light, stimulation time, and the interaction among these factors. Pupillary light reflex was considered a dependent variable, circadian time, type of light, and stimulation time as fixed factors, and animal as a random effect (test 2).

Daily profiles of $\tau$ data were compared by mixed-model statistical analysis to evaluate the effects of light type, circadian time, and the interaction among these factors. The model considered animal as random effects (test 3). Profiles of $\tau$ data were natural logarithm-transformed for normality of residuals.

The pupillary light reflexes data of the SP were compared by mixed-model statistical analysis to evaluate the effects of circadian time, type of light, and interaction among these factors. Stationary PLR was considered a dependent variable, circadian time and type of light as fixed factors, and animal as a random effect (test 4). To model the heteroscedasticity, an identity function of variance was incorporated (including the type of light covariable in the function).

The Fisher least significant difference test was used for posthoc analysis. Differences were considered significant at $P<$ 0.05 . All values are expressed as mean \pm SEM.

\section{Results}

\section{GUCY1* Chickens Exhibit Daily Changes in the PLR}

The pupillary light reflexes of GUCY1* chickens was significantly influenced by white light or blue light of $475 \mathrm{~nm}$ in combination with circadian time (Test $1, F_{16,268}=2.6, P<$ 0.01 , Fig. 1), or with stimulation time (Test $1, F_{8}, 268=4.78, P$ $<0.01$, Fig. 1). When white light was used as stimulus (Fig. 1, upper), the PLR was significantly different between subjective day (circadian time [CT]3: $57.37 \pm 3.29$; CT6: $55.26 \pm 3.29$ ) and subjective night (CT18: $66.12 \pm 3.29$; CT21: $72.30 \pm 3.29$; CT24: $68.77 \pm 3.29)$. Also, as observed with white light, with blue light stimuli of $475 \mathrm{~nm}$ (Fig. 1, bottom), the PLR shows statistically different daily variations between subjective day (CT6: $45.3 \pm 3.29$ ) and subjective night (CT18: $59.74 \pm 3.29$ ). When blue light of $430 \mathrm{~nm}$ was used as a control (Fig. 1, middle), the PLR did not differ significantly between subjective day (CT3: $66.85 \pm 3.29$; CT6: $74.53 \pm 3.29$ ) and subjective night (CT18: $66.78 \pm 3.29$; CT21: $72.80 \pm 3.29$, respectively) although the light intensity applied was lower.

Since the PLR has two clearly distinguishable phases, the NSP and SP, which provide different but complementary information (see Materials and Methods), we sought to assess

variation of PLR with bright white light. Middle: pupillary light reflexes with blue light of $430 \mathrm{~nm}$. Bottom: circadian variation of PLR with blue light of $475 \mathrm{~nm}$. (B) Differences between different types of light at CT 6 and CT 21. Results are presented as percentage of maximum linear pupil constriction. Data are means \pm SEM; $n=3$. Statistical analysis revealed a significant effect of time of day for white and blue light ( $475 \mathrm{~nm} ; P \leq 0.0001)$. 


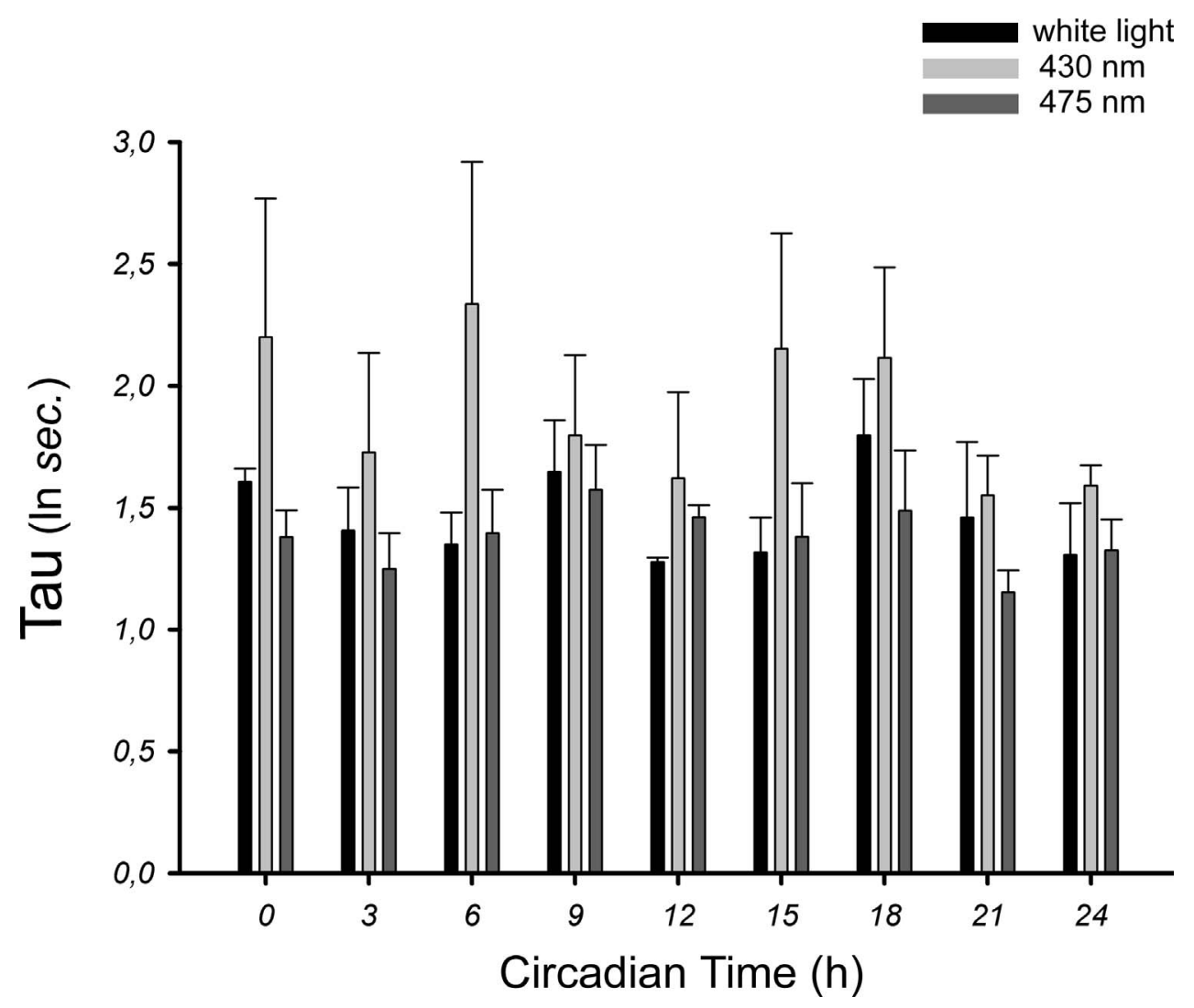

Figure 3. Pupillary light reflex kinetics exhibits no changes throughout the day (test 3). Daily profiles of the mean $\tau$ for white (black bars), 430(light gray bars) and $475-\mathrm{nm}$ blue light (dark gray bars) stimuli. Statistical analysis revealed a significant effect of light type $(P=0.003)$ : PLR lifetimes obtained after a 430-nm light stimulus were significantly longer than those obtained with white or 475-nm blue light. Natural logarithmtransformed are means $\pm \mathrm{SEM} ; n=3$.

whether the observed PLR differences arise from differences in the NSP, in the SP, or in both. Therefore, we conducted a separated analysis of our results for these two phases.

\section{Analysis of the Nonstationary PLR With $475 \mathrm{~nm}$ and White Lights}

The nonstationary phase of PLR was significantly influenced by white or 475-nm lights in combination with circadian time (Test $2, F_{16,376}=4.67, P<0.01$, Fig. 2), or with stimulation time (Test $2, F_{12,376}=3.55, P<0.01$, Fig. 2). When white light was used as stimulus (Fig. 2A, upper, 2B), the PLR exhibited daily changes, with maximum values during the subjective day (CT6: $53.39 \pm 3.20$ ) and minimum during the subjective night (CT21: 70.53 \pm 3.20 ). Daily changes in the pupillary responses were also observed with blue light stimuli of $475 \mathrm{~nm}$ and the control of $430 \mathrm{~nm}$; however, no clear differences between subjective day and night (Fig. 2, middle and bottom) were observed. Figure 2B shows the percentage of constriction at CT6 and CT21 for each light condition in order to highlight the differences between subjective day and subjective night in the NSP of the PLRs. At CT6, the pupillary constriction responses in GUCY1* chickens displayed the highest responses with white or blue light of $475 \mathrm{~nm}$ (Fig. 2B). At circadian time 21, however, the pupillary responses stimulated with white light were similar to the controls stimulated with blue light of 430 $\mathrm{nm}$, but differed markedly from those obtained with blue light of $475 \mathrm{~nm}$ (Fig. 2B).

\section{PLR Kinetics Does Not Exhibit Changes Throughout the Day}

The daily differences observed during the NSP of the PLR driven by the white light stimulus led us to ask whether or not there are daily changes in the PLR kinetics of GUCY1* chickens. We therefore sought to determine the characteristic lifetime $(\tau, \mathrm{sec})$ for the PRL time courses throughout the day. To this end, we fitted the PLR time courses for each chicken at each light type and CT condition with a first-order exponential decay function to obtain their lifetimes. Figure 3 shows that there were no daily changes in the PLR kinetics of GUCY1* chickens for the conditions tested.

\section{Analysis of the Stationary PLR}

Stationary PLR data were significantly influenced by white or 475 -nm lights in combination with circadian time $\left(F_{16,295}=\right.$ 2.91, $P<0.01$, test 4 ). The largest percentage of constriction values with white light were observed during the subjective day (CT3: $47.42 \pm 3.57$, СT6: $45.79 \pm 3.57$ ), while the lowest values were observed during the subjective night (CT21: 65.84 \pm 3.57, CT24: 63.12 \pm 3.57; Fig. 4A, upper, 4B). Daily variations were observed in consensual pupillary light reflex with blue light of $475 \mathrm{~nm}$ (Fig. 4A, bottom). The maximum percentage of constriction was observed during the subjective day (CT6: $32.45 \pm 2.91$, CT9: $36.74 \pm 2.91$ ), while the minimal values were observed during the subjective night (CT18: $47.97 \pm 2.91$, CT21: $44.45 \pm 2.91$ ). No diurnal variations were observed in the controls with blue light of 430 
A
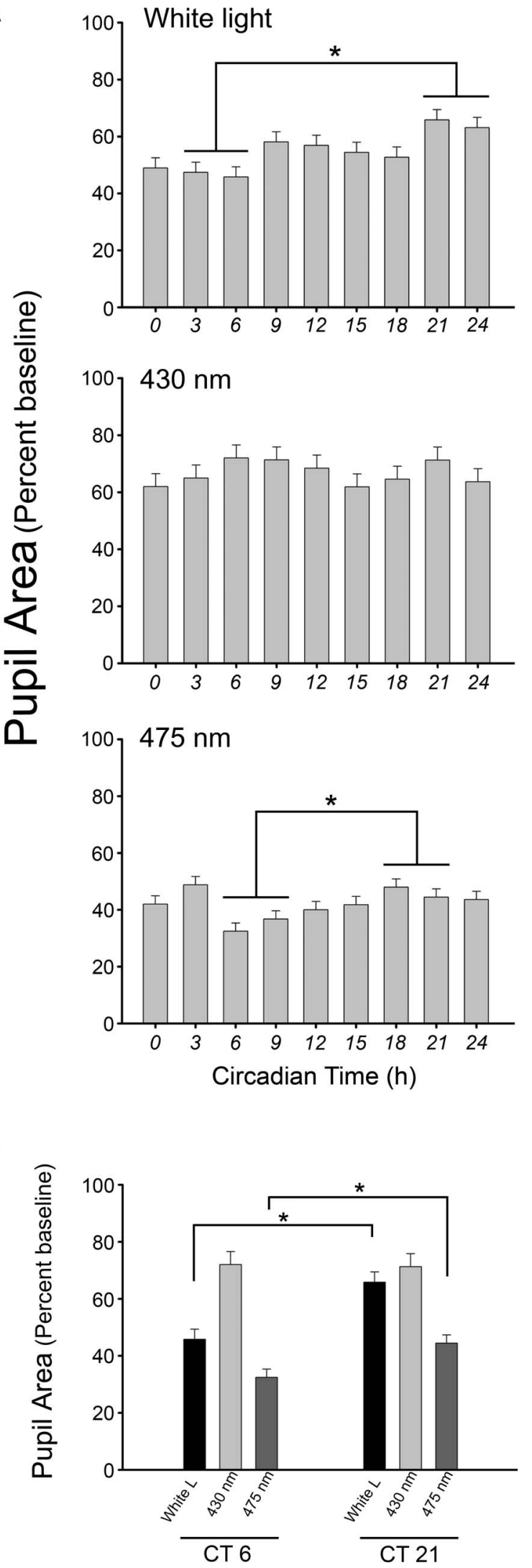

FIGURE 4. Analysis of the stationary pupillary light responses in GUCY1* chickens exposed to different types of light under scotopic conditions. (A) Circadian pupillary light response at Stationary phase (18-32 seconds of stimulation) in GUCY1* chickens exposed to nm $(P>0.05$; Fig. 4A, middle), nor did the values differ from those observed for white light at CT21 (Fig. 4B). In addition, although the light intensities used for the blue lights (430/475 $\mathrm{nm}$ ) were different, values with blue light of $475 \mathrm{~nm}$ were lower than those observed with the controls exposed to 430nm light.

\section{Discussion}

In the present work we demonstrate a circadian rhythm in consensual PLRs in a nonmammalian model of blindness, the GUCY1* chickens, kept under constant illumination conditions (DD). These birds carry an autosomal recessive mutation in the photoreceptor-specific guanylate cyclase 1 (GC1) gene ${ }^{17}$ that severely affects both phototransduction and survival of cones and rods. ${ }^{18,19}$ This retinopathy causes blindness at hatch, similar to Leber's congenital amaurosis in humans. ${ }^{20}$ However, these birds still retain the ability to detect light that regulates a variety of nonimage forming functions such as the photic entrainment of feeding rhythms and PLRs. ${ }^{6,12}$ Furthermore, as previously shown in WT RGCs, ${ }^{21}$ in GUCY1* birds these cells are still able to give circadian expression to the mRNA for aralkylamine $\mathrm{N}$-acetyltransferase (AA-NAT), a key regulatory enzyme in the synthesis of melatonin, albeit with a completely shifted phase. ${ }^{22}$

In most studies on PLRs, pupil amplitude (either absolute or relative to the starting pupil diameter) is the main parameter for characterizing pupil constriction in response to light. 1,3,23,24,27 However, other variables could also be considered, including latency, velocity, or acceleration. ${ }^{25,26}$

Here we have examined two of these PLR variables in GUCY1* chickens: the relative amplitude (\% constriction) and velocity (kinetics) of the responses. A clear daily change was visualized in pupil amplitude during white light stimulation (Fig. 1); this amplitude variation was observed in both the NSP (Fig. 2A) and the SP (Fig. 4A) of the PLR, indicating that both phases contribute to the whole response and that the daily control of pupil size is measurable after only a few seconds of the bright white light stimulus onset.

Similar daily changes in whole PLR amplitude were observed in response to a 475 -nm blue light stimulus (12.05 $\mathrm{mW} / \mathrm{cm}^{2}$; Fig. 1), but in this case the changes appear to be entirely due to differences observed during the SP (Fig. 4B), since no clear daily differences were seen during the NSP (Fig. 2). Thus, with a bright $475-\mathrm{nm}$ blue light stimulus, the daily control of PRL would seem to be measurable only when the pupil has reached its stable minimum size.

As expected, levels of constriction during the SP for a 475$\mathrm{nm}$ blue light pulse are higher than those for a white light pulse (Fig. 4B) since the opsin photopigment driving the PLRs in GUCY $1^{*}$ birds is most sensitive around $484 \mathrm{~nm}$. This also explains why, when using a $475-\mathrm{nm}$ blue light stimulus, the percentage of constriction obtained during the NSP throughout the circadian times (Fig. 2A) was more pronounced than that observed with a white light stimulus at both subjective day and night (Figs. 2A, 2B). By contrast, no daily differences in the PLR amplitude under a 430-nm light stimulus $(0.35 \mathrm{~mW} /$

different types of light under scotopic conditions (test 4). Upper: circadian variation of PLR with bright white light. Middle: pupillary light reflex with blue light of $430 \mathrm{~nm}$. Bottom: circadian variation of PLR with blue light of $475 \mathrm{~nm}$. (B) Differences between different types of light at CT6 and CT21. Results are presented as percentage of maximum linear pupil constriction. Data are means $\pm \mathrm{SEM} ; n=3$. Statistical analysis revealed a significant effect of time of day for white and blue light $(475 \mathrm{~nm})(P \leq 0.0001)$. 
$\mathrm{cm}^{2}$ ) were found after the whole statistical analysis (Fig. 1, middle) or the analysis of the SP and NSP conducted separately (Figs. 2, 4, middle). Nevertheless, a small percentage of pupil constriction was observed in the controls exposed to a 430-nm blue light stimulus (at both SP and NSP). It is worth noting here that although the intensity of the $430-\mathrm{nm}$ light stimulus used in the experiments produces a $40 \%$ to $50 \%$ maximum constriction (see the 430-nm dose-response curve in Ref. 6, Fig. 3), we cannot completely rule out the possibility that circadian control of the PLR amplitude might occur at higher intensities for this wavelength. Furthermore, because the 475 and $430 \mathrm{~nm}$ lights used here were not equiluminent, we cannot directly compare their daily profiles. Nevertheless, the comparisons between circadian phases for the same light sources (white or $475 \mathrm{~nm}$ ) clearly demonstrates a circadian control of the PLR in GUCY1* chickens.

We have previously determined the dose-response curves and action spectrum for the PLR in GUCY1* chickens, ${ }^{6}$ which revealed that an opsin-based photopigment, with maximum absorption at $484 \mathrm{~nm}$, mediates these responses. In addition, we have also demonstrated that the inner retina of GUCY1* chickens expresses the two melanopsin genes (Opn $4 x$ and Opn $4 \mathrm{~m}$ ) as well as their corresponding proteins. ${ }^{6,15}$ These observations, together with the fact that melanopsin is expressed in a circadian manner with higher levels during the light phase in chicken retinal cells, ${ }^{34}$ suggest that the circadian PLR shown in this work could be driven by opsin 4 . Cryptochromes, which display maximal absorbance at $430 \mathrm{~nm}$, have been associated with some nonvisual photoperception tasks at early developmental stages in chickens, though these responses disappear after birth.6,28 Moreover, our previous studies suggest that cryptochromes do not act as functional photopigments directly responsible for conducting the PLRs in adult GUCY1* chickens. ${ }^{6}$ Nevertheless, we cannot completely rule out this possibility on the basis of the results shown here.

Despite the white and 475-nm blue light stimuli not being equiluminent, the kinetic responses obtained were similar at any CT examined, reflecting that both light conditions were equally efficient for driving daily variations in the PLRs.

Our results also suggest that under the light conditions presented in this work, there are no daily changes in the slope $(\tau)$ of the PLR time courses for GUCY1* chickens, despite $\tau_{s}$ being calculated via different fitting approaches. ${ }^{29}$ As we mentioned above, there is indeed a daily rhythm in the pupil amplitude during the NSP when the stimulus was white light. A parsimonious explanation for these observations could be that both phases contribute to the whole PLR under white light, reflecting the fact that the amplitude of the whole PLR time courses for some CTs were higher than others, maintaining the slopes.

It is therefore likely that the small PLR observed with a 430$\mathrm{nm}$ light stimulus is because the light source used for these experiments exhibited an emission spectrum peaking at 430 $\mathrm{nm}$, but with a tail overlapping the absorption spectrum for melanopsin (Supplementary Fig. S1). This observation also explains why the characteristic lifetime $(\tau)$ for the PRL time courses under a 430-nm light stimulus are longer and the PLRs slower (Fig. 3) than those obtained under the white and 475$\mathrm{nm}$ blue light conditions. In this connection, under white or 475-nm blue light stimuli, the kinetic responses did not differ statistically at any CT examined, reflecting that the two light pulses are equally efficient at driving the PLRs.

Another interesting finding is that GC1 is not essential for the functioning of the photocascade operating in nonclassical photoreceptors located in the inner retina. This strongly suggests that the biochemical photocascade operating in the nonvisual inner retinal photoreceptors does not require the synthesis of cGMP by GC1 as occurs in isolated ipRGCs. ${ }^{30-32}$ In the chicken and mammalian retina, GC2 (also known as GC-F) is also expressed; however, levels of cGMP in GUCY1* chicken retinas are 6-fold lower than those in WT animals. ${ }^{17}$

It is known that the chicken retina works as an autonomous oscillator independent of the suprachiasmatic nuclei, displaying circadian rhythms in melatonin, dopamine, and photopigments synthesis, variation in the amplitude of electroretinogram components, and glycerophospholipid synthesis-among others $^{21,22,33-41}$-reviewed in Guido et al. ${ }^{42}$ Moreover, we have previously shown that chicken RGCs also contain autonomous oscillators capable of generating self-sustained rhythms in melatonin synthesis and in the expression and activity of AANAT with higher levels during the day under DD, LL, or regular LD cycles. ${ }^{21,22,31}$ Strikingly, a number of activities controlled by retinal clocks presumably located in the RGCs were shown to peak during the day, such as the highest sensitivity to white and blue $(475 \mathrm{~nm})$ light for the PLRs described in this study and specific ERG parameters: the b-wave amplitude and implicit time which were also higher during the day, ${ }^{37}$ when RGC melatonin peaks. ${ }^{21,22,31}$

These facts lead us to believe that our results, showing daily changes in pupillary responses under constant dark conditions, reflect truly circadian control of this nonimage photic response in GUCY1* chickens. Recently, a daily rhythm in PLR was also reported in the retinal degenerate mice $\mathrm{rd} / \mathrm{rd}$. However, these experiments were performed under a 12-hour light/12-hour dark cycle $^{2}$ so that a light-driven PLR rhythm cannot be completely excluded. Nevertheless, this PLR rhythm, together with the fact that clock mutant backgrounds reduced the PLR sensitivity of $\mathrm{rd} / \mathrm{rd}$ mice, strongly suggests the circadian modulation of PLR in mice. ${ }^{2}$

On the basis of previous results found in the literature, we suggest that at least two independent mechanisms could be involved in the circadian modulation shown here. The first is at the level of the retina itself, since the ipRGCs involved in the control of pupil size may act as independent oscillators controlling this response in humans. ${ }^{14}$ Furthermore, the melanopsin photopigment in the chicken inner retina is expressed in a circadian manner with higher levels during the subjective day, ${ }^{34}$ regulated by dopamine ${ }^{43}$ that, in turn, also exhibit a daily rhythm with higher levels during the light phase. ${ }^{40,41}$ We have previously demonstrated that GUCY1* inner retinas express the two genes of melanopsin (Opn $4 x$ and Opn $4 m$ ) at the mRNA and protein levels ${ }^{6,15}$ and here we observed stronger pupillary responses during the subjective day than the subjective night.

The second mechanism could be indirect circadian control exerted by the suprachiasmatic nuclei on the Edinger-Westphal nuclei, which are critical in controlling pupil size. ${ }^{44}$

Overall, our results clearly demonstrate for the first time the circadian control of the PLRs in nonmammalian vertebrates with no functional cones or rods (GUCY1* chickens) and support the hypothesis that the circadian control of PLR may occur in these blind animals, mediated by a vitamin A-based photopigment with a maximum sensitivity around $480 \mathrm{~nm}$.

\section{Acknowledgments}

Supported by the Agencia Nacional de Promoción Científica y Técnica (Fondo para la Investigación Científica y Tecnológica; PICT 2004 no. 967, PICT 2006 no. 898, PICT Bicentenario 2010 no. 647); Consejo Nacional de Investigaciones Científicas y Técnicas de la República Argentina; Ministerio de Ciencia y Tecnología de la Provincia de Córdoba; and Secretaría de Ciencia y Técnica-Universidad Nacional de Córdoba.

Disclosure: D.J. Valdez, None; P.S. Nieto, None; N.S. Della Costa, None; C. Schurrer, None; M.E. Guido, None 


\section{References}

1. Lucas RJ, Hattar S, Takao M, Berson DM, Foster RG, Yau KW. Diminished pupillary light reflex at high irradiances in melanopsin-knockout mice. Science. 2003;299:245-247.

2. Owens L, Buhr E, Tu DC, Lamprecht TL, Lee J, Van Gelder RN. Effect of circadian clock gene mutations on nonvisual photoreception in the mouse. Invest Ophthalmol Vis Sci. 2012;53:454-460.

3. Panda S, Provencio I, Tu DC, et al. Melanopsin is required for non-image-forming photic responses in blind mice. Science. 2003;301:525-527.

4. Rollag MD, Berson DM, Provencio I. Melanopsin, ganglion-cell photoreceptors, and mammalian photoentrainment. $J$ Biol Rhythms. 2003;18:227-234.

5. Semo M, Vugler AA, Jeffery G. Paradoxical opsin expressing cells in the inner retina that are augmented following retinal degeneration. Eur J Neurosci. 2007;25:2296-2306.

6. Valdez DJ, Nieto PS, Garbarino-Pico E, et al. A nonmammalian vertebrate model of blindness reveals functional photoreceptors in the inner retina. FASEB J. 2009;23:1186-1195.

7. Provencio I, Rodriguez IR, Jiang G, Hayes WP, Moreira EF, Rollag MD. A novel human opsin in the inner retina. $J$ Neurosci. 2000;20:600-605.

8. Hattar S, Liao HW, Takao M, Berson DM, Yau KW. Melanopsincontaining retinal ganglion cells: architecture, projections, and intrinsic photosensitivity. Science. 2002;295:1065-1070.

9. Lucas RJ, Foster RG. Neither functional rod photoreceptors nor rod or cone outer segments are required for the photic inhibition of pineal melatonin 1. Endocrinology. 1999;140: 1520-1524.

10. Lucas RJ, Freedman MS, Muñoz M, Garcia-Fernández J-M, Foster RG. Regulation of the mammalian pineal by non-rod, non-cone, ocular photoreceptors. Science. 1999;284:505-507.

11. Semo M, Peirson S, Lupi D, Lucas RJ, Jeffery G, Foster RG. Melanopsin retinal ganglion cells and the maintenance of circadian and pupillary responses to light in aged rodless/ coneless (rd/rd cl) mice. Eur J Neurosci. 2003;17:1793-1801.

12. Valdez DJ, Nieto PS, Diaz NM, Garbarino-Pico E, Guido ME. Differential regulation of feeding rhythms through a multiplephotoreceptor system in an avian model of blindness. FASEB J. 2013;27:2702-2712.

13. Munch M, Leon L, Crippa SV, Kawasaki A. Circadian and wakedependent effects on the pupil light reflex in response to narrow-bandwidth light pulses. Invest Ophthalmol Vis Sci. 2012;53:4546-4555.

14. Zele AJ, Feigl B, Smith SS, Markwell EL. The circadian response of intrinsically photosensitive retinal ganglion cells. PLOS ONE. 2011;6:e17860.

15. Verra DM, Contin MA, Hicks D, Guido ME. Early onset and differential temporospatial expression of melanopsin isoforms in the developing chicken retina. Invest Ophthalmol Vis Sci. 2011;52:5111-5120.

16. Di Rienzo J, Casanoves F, Balzarini M, Gonzalez L, Tablada M, Robledo C. InfoStat. 2011; Grupo InfoStat, FCA, Universidad Nacional de Córdoba, Argentina. Available at: http://www. infostat com.ar. Accessed November 2, 2014.

17. Semple-Rowland SL, Lee NR, Van Hooser JP, Palczewski K, Baehr W. A null mutation in the photoreceptor guanylate cyclase gene causes the retinal degeneration chicken phenotype. Proc Natl Acad Sci U S A. 1998;95:1271-1276.

18. Ulshafer RJ, Allen C, Dawson WW, Wolf ED. Hereditary retinal degeneration in the Rhode Island Red chicken. I. Histology and ERG. Exp Eye Res. 1984;39:125-135.

19. Ulshafer RJ, Allen CB. Scanning electron microscopy of the retina in an animal model of hereditary blindness. Scan Electron Microsc. 1984;841-848.
20. Hanein S, Perrault I, Gerber S, et al. Leber congenital amaurosis: comprehensive survey of the genetic heterogeneity, refinement of the clinical definition, and genotypephenotype correlations as a strategy for molecular diagnosis. Hum Mutat. 2004;23:306-317.

21. Garbarino-Pico E, Carpentieri AR, Contin MA, et al. Retinal ganglion cells are autonomous circadian oscillators synthesizing N-acetylserotonin during the day. J Biol Chem. 2004;279: 51172-51181.

22. Valdez DJ, Garbarino-Pico E, Diaz NM, Silvestre DC, Guido ME. Differential regulation of arylalkylamine $\mathrm{N}$-acetyltransferase activity in chicken retinal ganglion cells by light and circadian clock. Chronobiol Int. 2012;29:1011-1020.

23. Lucas RJ, Douglas RH, Foster RG. Characterization of an ocular photopigment capable of driving pupillary constriction in mice. Nat Neurosci. 2001;4:621-626.

24. Peirson SN, Thompson S, Hankins MW, Foster RG. Mammalian photoentrainment: results, methods, and approaches. Methods Enzymol. 2005;393:697-726.

25. Bremner FD. Pupillometric evaluation of the dynamics of the pupillary response to a brief light stimulus in healthy subjects. Invest Ophthalmol Vis Sci. 2012;53:7343-7347.

26. Clarke RJ, Zhang H, Gamlin PD. Characteristics of the pupillary light reflex in the alert rhesus monkey. J Neurophysiol. 2003; 89:3179-3189.

27. Hattar S, Lucas RJ, Mrosovsky N, et al. Melanopsin and rodcone photoreceptive systems account for all major accessory visual functions in mice. Nature. 2003;424:76-81.

28. Tu DC, Batten ML, Palczewski K, Van Gelder RN. Nonvisual photoreception in the chick iris. Science. 2004;306:129-131.

29. Motulsky H. Fitting Models to Biological Data Using Linear and Nonlinear Regression: a Practical Guide to Curve Fitting: A Practical Guide to Curve Fitting. Bethesda: Oxford University Press; 2004.

30. Bailes HJ, Lucas RJ. Human melanopsin forms a pigment maximally sensitive to blue light (lambdamax approximately $479 \mathrm{~nm})$ supporting activation of $\mathrm{G}(\mathrm{q} / 11)$ and $\mathrm{G}(\mathrm{i} / \mathrm{o})$ signalling cascades. Proc Biol Sci. 2013;280:20122987.

31. Contin MA, Verra DM, Guido ME. An invertebrate-like phototransduction cascade mediates light detection in the chicken retinal ganglion cells. FASEB J. 2006;20:2648-2650.

32. Contin MA, Verra DM, Salvador G, Ilincheta M, Giusto NM, Guido ME. Light activation of the phosphoinositide cycle in intrinsically photosensitive chicken retinal ganglion cells. Invest Ophthalmol Vis Sci. 2010;51:5491-5498.

33. Bailey MJ, Cassone VM. Melanopsin expression in the chick retina and pineal gland. Brain Res Mol Brain Res. 2005;134: 345-348.

34. Chaurasia SS, Rollag MD, Jiang G, et al. Molecular cloning, localization and circadian expression of chicken melanopsin (Opn4): differential regulation of expression in pineal and retinal cell types. J Neurochem. 2005;92:158-170.

35. Garbarino-Pico E, Carpentieri A, Castagnet P, et al. Synthesis of retinal ganglion cell phospholipids is under control of an endogenous circadian clock: Daily variations in phospholipidsynthesizing enzyme activities. J Neurosc Res. 2004;76:642-652.

36. Garbarino-Pico E, Valdez DJ, Contín MA, et al. Rhythms of glycerophospholipid synthesis in retinal inner nuclear layer cells. Neurochem Int. 2005;47:260-270.

37. McGoogan JM, Cassone VM. Circadian regulation of chick electroretinogram: effects of pinealectomy and exogenous melatonin. Am J Physiol. 1999;277:R1418-R1427.

38. Nir I, Haque R, Iuvone PM. Diurnal metabolism of dopamine in dystrophic retinas of homozygous and heterozygous retinal degeneration slow (rds) mice. Brain Res. 2000;884:13-22.

39. Tomonari S, Takagi A, Akamatsu S, Noji S, Ohuchi H. A noncanonical photopigment, melanopsin, is expressed in the 
differentiating ganglion, horizontal, and bipolar cells of the chicken retina. Dev Dyn. 2005;234:783-790.

40. Zawilska JB, Bednarek A, Berezinska M, Nowak JZ. Rhythmic changes in metabolism of dopamine in the chick retina: the importance of light versus biological clock. J Neurochem. 2003;84:717-724.

41. Zawilska JB, Berezinska M, Rosiak J, Vivien-Roels B, Nowak JZ. The relationship between melatonin and dopamine rhythms in the duck retina. Neurosci Lett. 2003;347:37-40.

42. Guido ME, Garbarino-Pico E, Contin MA, et al. Inner retinal circadian clocks and non-visual photoreceptors: novel players in the circadian system. Prog Neurobiol. 2010;92: 484-504.

43. Sakamoto K, Liu C, Kasamatsu M, Pozdeyev NV, Iuvone PM, Tosini G. Dopamine regulates melanopsin mRNA expression in intrinsically photosensitive retinal ganglion cells. Eur $J$ Neurosci. 2005;22:3129-3136.

44. Cantwell EL, Cassone VM. Chicken suprachiasmatic nuclei: I. Efferent and afferent connections. J Comp Neurol. 2006;496: 97-120. 